\title{
Systematic differences in the spectroscopic analysis of red giants $\star, \star \star$
}

\author{
D. Slumstrup, F. Grundahl, V. Silva Aguirre, and K. Brogaard
}

\begin{abstract}
Stellar Astrophysics Centre, Department of Physics and Astronomy, Aarhus University, Ny Munkegade 120, 8000 Aarhus C, Denmark e-mail: ditte@phys. au.dk
\end{abstract}

Received 29 June 2018 / Accepted 11 December 2018

\begin{abstract}
Aims. A spectroscopic analysis of stellar spectra can be carried out using multiple approaches such as different methods, line lists, atmospheric models, atomic parameters, and solar abundances. The resulting atmospheric parameters from these choices can vary beyond the quoted uncertainties in the literature. We characterize these differences by systematically comparing some of the commonly adopted ingredients such as line lists, equivalent width measurements, and atomic parameters.

Methods. High-resolution and high signal-to-noise $(\mathrm{S} / \mathrm{N})$ spectroscopic data of one helium-core-burning red giant star in each of the three open clusters, NGC 6819, M67, and NGC 188 were obtained with the FIES spectrograph at the Nordic Optical Telescope. The M67 target was used to benchmark the analysis, as it is a well-studied cluster with asteroseismic data from the K2 mission. For the other two clusters we obtained higher quality data than had been analyzed before, which allows us to establish their chemical composition more securely. Using a line by line analysis, we tested several different combinations of line lists and programs to measure equivalent widths of stellar absorption lines to characterize systematic differences within the same spectroscopic method.

Results. The obtained parameters for the benchmark star in M67 vary up to $\sim 170 \mathrm{~K}$ in effective temperature, $\sim 0.4 \mathrm{dex}$ in $\log g$ and $\sim 0.25 \mathrm{dex}$ in $[\mathrm{Fe} / \mathrm{H}]$ between the tested setups. Using the combination of an equivalent width measurement program and line list that best reproduce the inferred surface gravity from asteroseismology, we determined the atmospheric parameters for the three stars and securely established the chemical composition of NGC 6819 to be close to solar, $[\mathrm{Fe} / \mathrm{H}]=-0.02 \pm 0.01 \mathrm{dex}$.

Conclusions. We highlighted the significantly different results obtained with different combinations of line lists, programs, and atomic parameters. The results emphasize the importance of benchmark stars studied with several methods to anchor spectroscopic analyses.
\end{abstract}

Key words. stars: fundamental parameters - stars: abundances - open clusters and associations: individual: NGC 6819 open clusters and associations: individual: M67 - open clusters and associations: individual: NGC 188 - stars: late-type

\section{Introduction}

Several large spectroscopic surveys such as the Radial Velocity Experiment (RAVE; Steinmetz et al. 2006), the Apache Point Observatory Galactic Evolution Experiment (APOGEE; Majewski et al. 2017), the Gaia-ESO survey (GES; Gilmore et al. 2012; Randich et al. 2013) and GALactic Archaeology with HERMES (GALAH; De Silva et al. 2015) are being carried out to better understand the structure, kinematics, and evolution of stars and our Galaxy. Massive amounts of data are obtained every day that require a swift, precise, and accurate analysis to support the scientific goals of the aforementioned surveys. To achieve this, pipelines have been constructed that analyze the data in a homogeneous way, for example, ASPCAP (García Pérez et al. 2016) for the case of APOGEE. These pipelines are normally tested against benchmark stars, which are stars that have been observed and analyzed by more than one method and have extremely high quality data. In the case of atmospheric parameters, examples of benchmark stars can be targets that have either abundances determined to a high level

\footnotetext{
${ }^{\star}$ Based on spectroscopic observations made with the Nordic Optical Telescope operated by NOTSA at the Observatorio del Roque de los Muchachos (La Palma, Spain) of the Instituto de Astrofísica de Canarias.

$\star \star$ Full Table A.1 is only available at the CDS via anonymous ftp to cdsarc.u-strasbg.fr $(130.79 .128 .5)$ or via http://cdsarc.u-strasbg.fr/viz-bin/qcat?]/A+A/622/A111
}

of precision (e.g., Jofré et al. 2014), effective temperatures measured by interferometry (e.g., Casagrande et al. 2014), and ideally an independent estimate of surface gravity as well. In the latter case, the advent of asteroseismology as a tool for Galactic archaeology has opened a new window of opportunity via its synergies with large spectroscopic surveys by providing precise measurements of $\log g$ for thousands of stars across the Milky Way (see, e.g., Miglio et al. 2013; Casagrande et al. 2016; Silva Aguirre et al. 2018; Pinsonneault et al. 2018, just to name a few examples).

Many methods are available for spectroscopic analyses of optical stellar spectra, varying from fitting synthethic spectra to observed spectra to classical equivalent width methods. For each of these methods, there are also different options for programs; for example Spectroscopy Made Easy (SME; Valenti \& Piskunov 1996), widely applied for synthetic fitting using a chi-square minimization algorithm and MOOG (Sneden 1973), is widely used for the classical equivalent width approach. It is well known that these different options do not always yield the same result and the extent of the differences can be significant. For a comparison of spectral synthesis methods, which we do not explore further in this work, see, for example, Lebzelter et al. (2012). For a comparison of equivalent width studies in the GES framework, see, for example, Smiljanic et al. (2014), where agreement between multiple determinations of atmospheric parameters for the same star are better than $82 \mathrm{~K}, 0.19 \mathrm{dex}$, and $0.10 \mathrm{dex}$ for $T_{\text {eff }}, \log g$, and $[\mathrm{Fe} / \mathrm{H}]$, respectively. For larger comparisons of 
both spectral synthesis methods and equivalent width methods, see, for example, Jofré et al. (2014) and Hinkel et al. (2016), where more detailed discussions on this and efforts to understand the causes of variations between stellar abundance measurement techniques can be found. Both studies have a list of stellar spectra analyzed with different methods and with and without restrictions on stellar parameters and line list. Casamiquela et al. (2017) studied the differences between spectral synthesis and equivalent widths by analyzing data of red clump stars in several open clusters and found an offset between the two methods in metallicity of $0.07 \pm 0.05$ dex. Moreover, the choice of absorption lines can highly affect the result. Even when choosing only lines that have the best measured atomic parameters and are not blended to any level of significance, strikingly different results in atmospheric parameters can be obtained; see, for example, Doyle et al. (2017) who find variations up to $50 \mathrm{~K}$. This paper also aims at highlighting this issue.

To perform an accurate spectroscopic analysis, it is necessary to know the level of systematic uncertainties incurred when a particular selection of, for example, the synthetic fitting method or equivalent width measurement program is made. This can be achieved by a detailed comparison of the results provided by these combinations when analyzing the same spectra if an empirical measurement of a stellar property exists as well. For example Smiljanic et al. (2014) and Casamiquela et al. (2017) used cluster membership as an independent constraint to evaluate the results. In this paper, we take one step further in this direction by not only including cluster studies but also asteroseismology in comparison with spectroscopic results obtained when varying one source of systematic uncertainty at a time.

In this paper, we chose the method of measuring equivalent widths of individual absorption lines and requiring excitation and ionization equilibria to hold when performing a differential analysis. We compare several combinations of line lists and programs to measure equivalent widths to understand the systematic uncertainties within the line by line spectroscopic analysis.

We carry out a detailed fundamental parameter and abundance analysis of three targets, one in each of the three open clusters (NGC 6819, M67, and NGC 188), based on high-resolution and high signal-to-noise (S/N) spectroscopic data from FIES (FIbre-fed Echelle Spectrograph) at the Nordic Optical Telescope (NOT). The targets have been selected to be in the same evolutionary phase (helium-core-burning) to make the analysis as homogeneous as possible. In the case of NGC 6819 and M67 there are also asteroseismic data available from the Kepler (Borucki et al. 1997) and K2 missions (Howell et al. 2014). Asteroseismology provides $\log g$ values with much higher precision than can normally be achieved with spectroscopy, which can greatly help constrain the spectroscopic analysis (see, e.g., Valentini et al. 2016; Pinsonneault et al. 2014, where asteroseismology has been used to calibrate the spectroscopic surface gravity by RAVE and APOGEE, respectively). It has however been shown that when doing a fully differential spectroscopic analysis of very high quality data, precision levels comparable to asteroseismology are achievable. Nissen (2015) did a differential analysis of solar twins to the Sun with very high quality spectra, $R=115000$ and $\mathrm{S} / \mathrm{N}$ levels above 600 , where an average uncertainty on the surface gravity of only 0.012 dex is obtained. This work was also compared and found to be in excellent agreement with a similar analysis by Ramírez et al. (2014) who achieved a surface gravity precision of 0.019 dex for spectra of $R=83000$ and $\mathrm{S} / \mathrm{N}$ levels above 400 . It is however important to note that this high level of precision is only achievable between stars of similar spectral type.
We chose stars in open clusters because they can be assumed to originate from the same molecular cloud and are therefore expected to have similar chemical composition and age. This also implies that by studying one confirmed member of a cluster, we get information about an entire population of stars. Open clusters have been the subject of extensive studies throughout the years, and therefore our results can be compared to literature values. This also has implications for other fields in astrophysics; clusters can be used as calibrators when studying galaxies, distances in the Universe, and age scales for stars. In order to exploit this fully, we need precisely determined properties of cluster stars involving different observing techniques to secure the accuracy.

The data used in our analysis for both NGC 6819 and NGC 188 is of higher quality (resolution and $\mathrm{S} / \mathrm{N}$ ) than previously used. To our knowledge, there is no high-resolution data $(R>65000)$ that also have $S / N>100$ for NGC 188 in the literature (see, e.g., Randich et al. 2003; Friel et al. 2010; Casamiquela et al. 2017 for previous studies of this cluster). Especially, the higher quality data allow us to better determine the metallicity of NGC 6819, on which there still is not a full consensus in the literature even though it is an otherwise very wellstudied cluster by asteroseismology because it is in the Kepler field. Previous determinations of the metallicity have been done by, for example, Bragaglia et al. (2001) and Lee-Brown et al. (2015) who analyzed three targets with $R \sim 40000$ yielding $[\mathrm{Fe} / \mathrm{H}]=0.09 \pm 0.03$ dex and 200 targets with $R \sim 13000$ yielding $[\mathrm{Fe} / \mathrm{H}]=-0.02 \pm 0.02$ dex, respectively.

\section{Observations}

We gathered data for confirmed members (Hole et al. 2009, Yadav et al. 2008, and Stetson et al. 2004) in the helium-coreburning phase, one in each of the three open clusters NGC 6819, M67, and NGC 188. M67 is a very well studied nearby solarlike cluster, which has also been observed with the $\mathrm{K} 2$ mission, and that target serves as a benchmark in the analysis. All targets are seen to be in the helium-core-burning evolutionary phase from color-magnitude diagrams (see Fig. 1). Futhermore, the two targets in M67 and NGC 6819 are also confirmed by asteroseismology to be in the helium-core-burning phase (Corsaro et al. 2012 and Stello et al. 2016).

The observations and data reduction are presented and described in more detail in Sect. 2 and Table A.1 of Slumstrup et al. (2017). Briefly, the spectra were obtained during period 47 and 51 with FIES at the NOT. The covered wavelength range is $3700 \AA$ and has high resolution $(R=67000)$ and high $\mathrm{S} / \mathrm{N}$ (above 100), which was estimated from the rms variation of the flux in a region around $6150 \AA$ for each spectrum. The coadded spectrum for each star was normalized order by order using RAINBOW ${ }^{1}$, which uses synthetic spectra with atmospheric parameters close to the expected for the target to guide the user to identify continuum points in the observed spectrum. To normalize the spectrum, the continuum points are then fitted with a spline function, which the continuum is then divided by. After this careful normalization, the orders are merged and the spectrum is shifted to laboratory wavelength to secure the line identification in the further analysis.

\section{Prior information}

As a starting point for the spectroscopic analysis, we use photometry and asteroseismology to give first guesses on effective

1 http://sites.google.com/site/vikingpowersoftware 

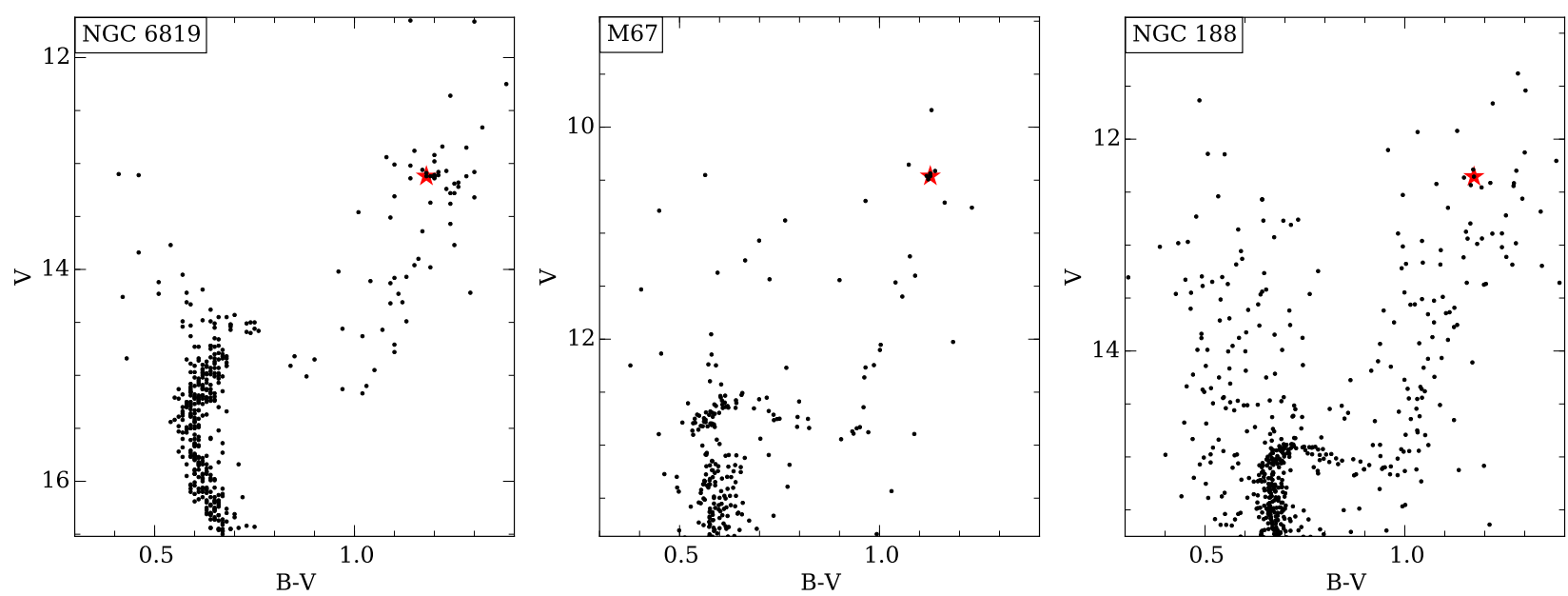

Fig. 1. From left to right panels: CMDs for NGC 6819 and M67 with photometry from Hole et al. (2009) and Yadav et al. (2008), respectively, and lastly a CMD for NGC 188 with photometry from the Stetson et al. (2004) standard fields. The target star for each cluster is marked with a red star.

Table 1. Temperatures from temperature-color calibrations.

\begin{tabular}{lcccccc}
\hline \hline Target & $B-V$ & $V-I$ & $V-K$ & $V-J$ & $V-H$ & Avg \\
\hline NGC6819 & 4749 & - & 4685 & 4659 & 4698 & $4698 \pm 33$ \\
M67 & 4643 & 4736 & 4697 & 4763 & 4749 & $4718 \pm 43$ \\
NGC188 & 4650 & 4610 & 4618 & 4651 & 4656 & $4637 \pm 19$ \\
\hline
\end{tabular}

temperature, $T_{\text {eff }}$, surface gravity, $\log g$, and metallicity, [Fe/H]. First guesses of effective temperature for the three targets were calculated with the metallicity dependent color-temperature relations by Ramírez \& Meléndez (2005), which are based on the infrared flux method. The optical photometry used is presented in Fig. 1 and the infrared photometry is from the 2MASS cata$\log$ (Skrutskie et al. 2006). The reddening values (presented in Table 1 of Slumstrup et al. 2017) used for these calculations are from Rosvick \& VandenBerg (1998), Taylor (2007), and Meibom et al. (2009) for NGC 6819, M67, and NGC 188, respectively. Results for each filter combination are presented in Table 1. The initial guesses for the temperatures were taken as averages of the various filter combinations for each target (last column of Table 1). The uncertainties on the photometric temperatures are only the scatter between the different color-temperature relations for each target and do not take into account the uncertainty in the relations themselves and should only be taken as a guide.

With the frequency of maximum power, $v_{\max }$ from asteroseismology and $T_{\text {eff }}$ from photometry, the surface gravity can be calculated using the scaling relation (Brown et al. 1991; Kjeldsen \& Bedding 1995),

$\log g=\log \left(\left(\frac{v_{\text {max }}}{3100}\right) \times\left(\frac{T_{\text {eff }}}{5777}\right)^{1 / 2}\right)+4.44$.

This relation is extrapolated from the Sun. Since we apply this relation for giants, deviations should be expected, which have been quantified in the literature with, for example, binary studies, parallaxes, and comparisons to interferometry. Asteroseismic masses have been shown to be accurate to better than $8 \%$ (Stello et al. 2016; Miglio et al. 2016; Brogaard et al. 2018) and radius better than 4\% (Huber et al. 2012, 2017; Silva Aguirre et al. 2012, 2016, White et al. 2013; Sahlholdt et al. 2018). Assuming a stellar mass of $1.2 M_{\odot}$ and radius of $10 R_{\odot}$, this translates to an accuracy on $\log g$ better than 0.04 dex. Even with only three months of data for the M67 target with K2, the precision is still better than what has been achieved for giants with normal spectroscopic methods in the literature that often suffer from systematic errors on the order of 0.2 dex (e.g., Bruntt et al. 2011; Thygesen et al. 2012; Heiter et al. 2015 to name a few); the exception to this is a strictly differential analyses described in the introduction. This provides the strongest constraint to the spectroscopic analysis. NGC 6819 is in the Kepler field with asteroseismic measurements available from extensive photometric time series (Corsaro et al. 2012; Handberg et al. 2017). M67 is in field 5 of the K2 mission, which also provides asteroseismology but with lower $\mathrm{S} / \mathrm{N}$ (Stello et al. 2016), as only three months of time series data have been acquired compared to the four year extent of the nominal Kepler mission.

There are no oscillation measurements available for NGC 188, but because there is a binary system near the main sequence turn-off in this cluster it is still possible to calculate an estimate of the surface gravity from photometry (Nissen et al. 1997) to a higher precision than for field stars with no strong mass constraints as follows:

$$
\begin{aligned}
\log \frac{g}{g_{\odot}}= & 4.44+\log \frac{M}{M_{\odot}}+4 \times \log \frac{T_{\mathrm{eff}}}{5777} \\
& +0.4 \times(V-3.1 E(B-V)) \\
& +0.4 \times \mathrm{BC}+2 \times \log \left(\frac{1}{d}\right)+0.12
\end{aligned}
$$

where $M$ is the mass. The quantity $M_{\mathrm{NGC} 188}=1.1 M_{\odot}$ is found by using a turn-off mass in the cluster of $1.1 M_{\odot}$ (Meibom et al. 2009) and assuming the evolution and mass loss after the turnoff advances as in NGC 6791, where the mass difference between the turn-off and red clump corresponds to the mass loss on the red giant branch (Brogaard et al. 2012; Miglio et al. 2012). The value $V$ is the $V$-band magnitude, $\mathrm{BC}$ is the bolometric correction, and $d$ is the distance calculated as

$d=10^{\left(1+\frac{\mu-3.1 \times E(B-V)}{5}\right)}$.

The apparent distance modulus in the $V$-band, $\mu=(m-M)_{V}$, is the difference between the apparent and absolute magnitude. The reddening and distance modulus for NGC 188 are from Meibom et al. (2009) and presented in Table 1 of Slumstrup et al. (2017). 
An empirical relation for the bolometric correction in Eq. (2) applicable for stars with $T_{\text {eff }}<5012 \mathrm{~K}$ is given in Torres (2010) as

$$
\mathrm{BC}=a+b\left(\log T_{\mathrm{eff}}\right)+c\left(\log T_{\mathrm{eff}}\right)^{2}+d\left(\log T_{\mathrm{eff}}\right)^{3},
$$

where $a, b, c, d$ are coefficients given in the article. The surface gravities from asteroseismology and photometry for the three targets are NGC 6819: $\log g=2.55 \pm 0.02$ dex, M67: $\log g=2.48 \pm 0.06 \mathrm{dex}$, and NGC 188: $\log g=2.45 \pm 0.14 \mathrm{dex}$.

\section{Comparison of methods}

To achieve a robust result, we did several realizations of the spectroscopic analysis using different combinations of line lists and equivalent width measurement programs and compared the outcome to determinations from asteroseismology and photometry. Because of the high accuracy of the asteroseismic $\log g$ as mentioned in Sect. 3, we used this as the main calibrator throughout the analysis. In the following section we describe the analysis carried out in detail, in which we used the M67 target as a benchmark and SPECTRUM (Gray \& Corbally 1994) to calculate atmospheric parameters from equivalent widths (see discussion in Sect. 5).

When asteroseismology is available the $\log g$ value can be held fixed in the spectroscopic analysis, which is often the chosen approach when possible. This can result in ionization equilibrium between FeI and FeII not being reached in the spectroscopic analysis. In most cases however this does not have a large impact on the effective temperature because excitation balance, which is used to obtain $T_{\text {eff }}$, depends on the excitation potentials and abundances of FeI lines, which are only mildly sensitive to pressure changes in the atmosphere.

Instead of fixing the $\log g$ value during our spectroscopic analysis, we used it as a reference when choosing our preferred combination of line list and equivalent width measurement program to make the spectroscopic results self consistent and in agreement with asteroseismology. Doyle et al. (2017) investigated the effect of fixing $\log g$ in the spectroscopic analysis of a set of FGK stars that already had accurate parameters determined from other methods. They found that fixing the surface gravity did not improve the precision on the other spectroscopic parameters, and found an average difference in determined $T_{\text {eff }}=3 \pm 13 \mathrm{~K}$. This is however in contrast to Hawkins et al. (2016) and Valentini et al. (2016) who both found better precision on the other atmospheric parameters when fixing the $\log g$ to the asteroseismic.

\subsection{Line lists}

To test the impact of choosing different combinations of absorption lines, we adopted one equivalent width measurement program (DAOSPEC, see discussion in Sect. 4.2). When choosing a line list, several considerations have to be made. The lines should not be blended, they should have well-determined atomic parameters, and they should cover a range of excitation potentials to get a robust effective temperature determination. Furthermore, the lines should preferably be weak enough to be on the linear part of their curve of growth (COG), yet strong enough to yield a significant determination, again covering a range of line strengths.

We ensured our lines were not blended by evaluating each individual transition for our range of atmospheric parameters with the online tool for the Vienna Atomic Line Database
(VALD; Piskunov et al. 1995). Based on our S/N values of 100-150, we decided that a possible blend should be stronger than $5 \%$ to be considered significant. This however only concerns atmospheric blends. A separate issue is that of telluric blends, which are in the rest frame of the Earth and can therefore not be evaluated with a tool like VALD. In order to avoid telluric blends, we chose to avoid parts of the spectrum with well-known telluric absorption lines.

We were careful in choosing absorption lines with only reliably determined atomic parameters, but there are still lines that do not yield the expected element abundances; for example, some Fe lines in the Sun do not give solar metallicity when using solar atmospheric parameters. This is the reason why we use the so-called astrophysical oscillator strengths, $\log g f$ values as an attempt to minimize effects from our lack of knowledge of atomic parameters. These are calibrated to the Sun by analyzing a solar spectrum taken with the same instrumental setup as the data and then adjusting the $\log g f$ values until each absorption line yields the expected solar chemical abundance. This can result in an increase in the precision of the metallicity by a factor of two when using calibrated values compared to laboratory values. It is however important to note that these values give more precise results by construction, but in doing so a bias can possibly be introduced in the analysis and care must be taken. Figure 2 shows this comparison for two sets of results for the M67 target using the same line list and program for measuring equivalent widths, where one set considers laboratory $\log g f$ values and the other astrophysical values. The kernel density estimator (KDE) plot on the right shows that not only is the scatter larger for the laboratory values ( $39 \mathrm{~K}$ compared to $18 \mathrm{~K}$ ), but also the resulting atmospheric parameters change: the chemical composition, effective temperature, and surface gravity increase when using laboratory values by $\Delta[\mathrm{Fe} / \mathrm{H}]=+0.05$, $\Delta T_{\text {eff }}=+100 \mathrm{~K}$, and $\Delta \log g=+0.27$, respectively (the difference between the two bottom results in Fig. 5). The value for the surface gravity of the M67 target is in poor agreement with the results from asteroseismology $\left(\log g_{\text {seis }}=2.48 \pm 0.06\right.$ as shown in Fig. 6), and photometry $\left(T_{\text {eff,phot }}=4718 \pm 43 \mathrm{~K}\right.$ as shown in Table 1). For the other two targets, NGC 6819 and NGC 188, the trend goes in the same direction but is more pronounced: $\Delta[\mathrm{Fe} / \mathrm{H}]=+0.02, \Delta T_{\text {eff }}=+160 \mathrm{~K}$ and $\Delta \log g=+0.35$ for NGC 188 and $\Delta[\mathrm{Fe} / \mathrm{H}]=+0.03, \Delta T_{\text {eff }}=+170 \mathrm{~K}$ and $\Delta \log g=+0.38$ for NGC 6819. We therefore chose to use the calibrated $\log g f$ values in our analysis. Doyle et al. (2017) found similar trends when comparing laboratory and solar calibrated $\log g f$ values, that $[\mathrm{Fe} / \mathrm{H}]$ is systematically higher with laboratory values, however their discrepancy on effective temperature is much smaller, $<10 \mathrm{~K}$.

As mentioned earlier in this section, the absorption lines preferably have to be weak enough to be on the linear part of the COG, which describes how the equivalent width of a line changes as the number of absorbers increase due to a change in a specific atmospheric parameter, such as the abundance of the element in question, $T_{\text {eff }}$ or $\log g$. For the three stars analyzed here, the linear part of the COG corresponds to lines with an equivalent width up to about $100 \mathrm{~m} \AA$. Figure 3 shows a comparison between equivalent width measurements of the same lines but with different programs (more on this in Sect. 4.2). Especially in the left plot of Fig. 3 it becomes apparent why stronger lines should be left out when possible: the scatter increases significantly for the stronger lines (around $\sim 90 \mathrm{~m} \AA$ and above). For instance, when including the stronger lines for the M67 target with astrophysical $\log g f$ values the atmospheric parameters 


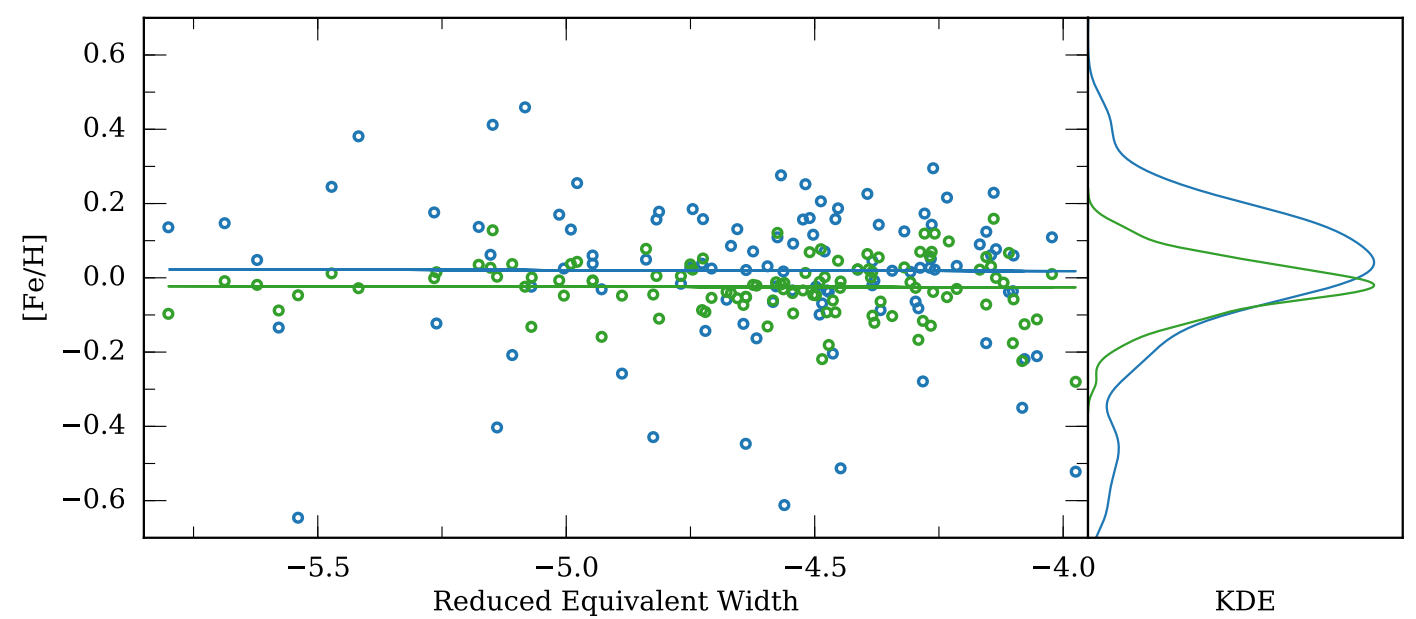

Fig. 2. Comparison of results using DAOSPEC and the line list based on Carraro et al. (2014) for laboratory (blue) vs. astrophysical (green) log $g f$ values for the M67 target. The right plot is a KDE illustrating the scatter in the two samples.
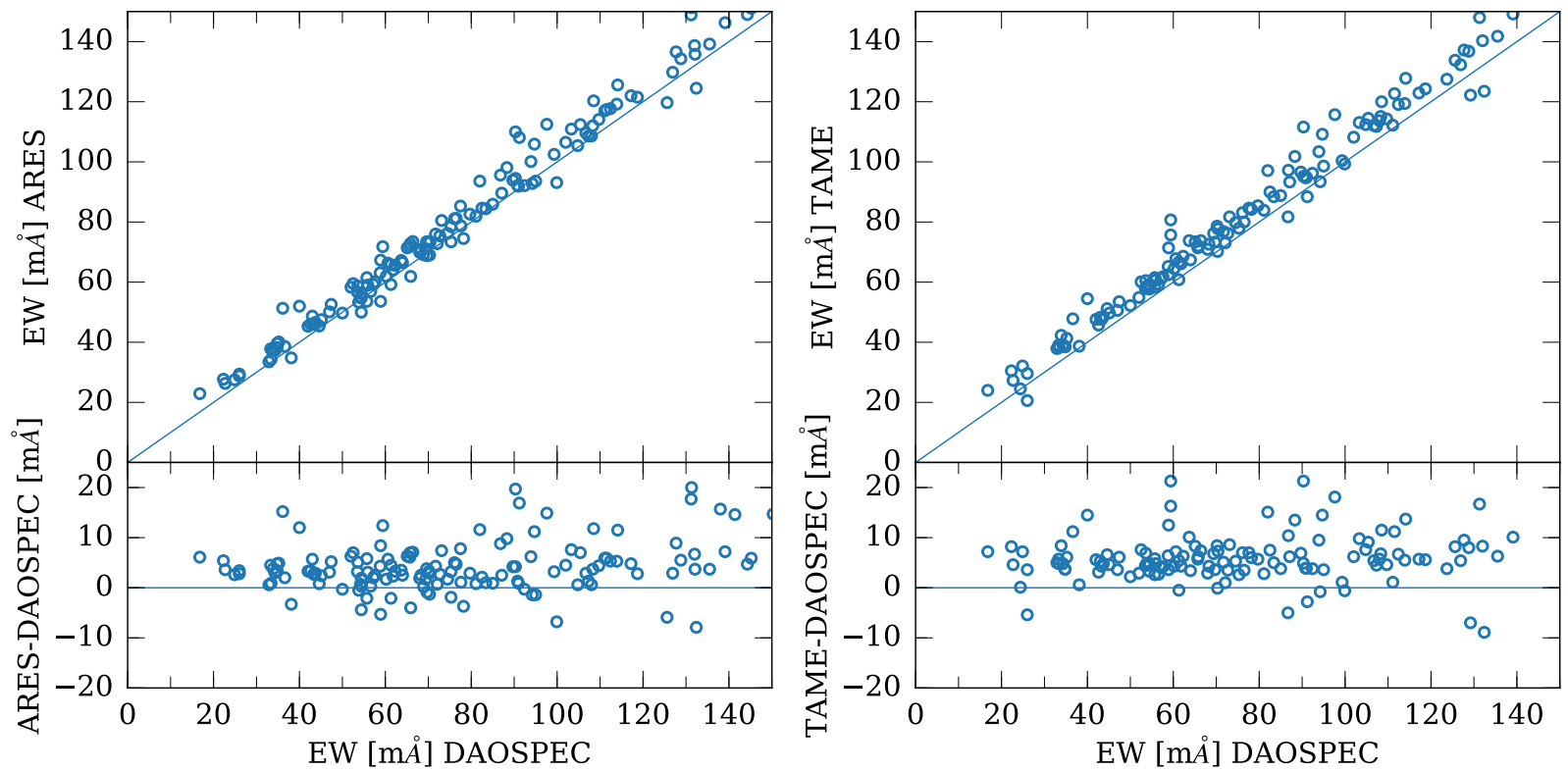

Fig. 3. Comparison of equivalent widths for the M67 target measured with three routines. Left panel: ARES vs. DAOSPEC with an average offset of $4.8 \mathrm{~m} \AA$. Right panel: TAME vs. DAOSPEC with an average offset of $5.9 \mathrm{~m} \AA$. In the spectroscopic analysis, Fe lines above $100 \mathrm{~m} \AA$ are discarded.

changed as well: $T_{\text {eff }}$ goes from 4680 to $4650 \mathrm{~K}\left(\Delta T_{\text {eff }}=-30 \mathrm{~K}\right)$, $\log g$ from 2.43 to $2.30 \operatorname{dex}(\Delta \log g=-0.13 \mathrm{dex})$ and $[\mathrm{Fe} / \mathrm{H}]$ from -0.03 to $0.00(\Delta[\mathrm{Fe} / \mathrm{H}]=0.03)$. For some elements with only a few good absorption lines in the optical part of the spectrum for a given atmospheric parameter range, the requirement of $100 \mathrm{~m} \AA$ can be difficult to reach. In our case, a few lines with higher equivalent widths than the $100 \mathrm{~m} \AA$ limit were used for elements other than Fe because of the lower number of lines available.

The higher the $\mathrm{S} / \mathrm{N}$ of the spectrum, the weaker the chosen lines can be because the depth of the line has to be significant compared to the noise level of the spectrum. Given our S/N levels, we set the lower limit at $10 \mathrm{~m} \AA$.

Golden lines. Jofré et al. (2014) collected results for a set of benchmark stars studied in the framework of the GES collaboration using many different pipelines to study systematic differences in the analysis. They created a list of "golden lines" (presented in Fig. 4), which are FeI and FeII lines all with well-determined atomic data that are on the linear part of the COG for all of their benchmark stars. These include FGK dwarfs and FGKM giants. Measurements of the strength of all the lines in the golden line list by several different groups agree within $2 \sigma$. Using only the golden lines for the FGK giants, the final scatter on the metallicity results from the different pipelines is around 0.08 dex. This is a good place to start when choosing lines and we tested this line list on the M67 target. It resulted in the atmospheric parameters $T_{\text {eff }}=4820 \mathrm{~K}$, $\log g=2.85 \mathrm{dex}$ and $[\mathrm{Fe} / \mathrm{H}]=-0.02 \mathrm{dex}$ (see Fig. 5, which shows the results from all the different combinations of line lists and programs considered in our study). The temperature is significantly higher than predicted from photometry (see Table 1), but the deciding part is the surface gravity that is much higher than the 2.48 dex predicted from asteroseismology. Even though an uncertainty on $\log g$ from spectroscopy of $0.2-0.3$ dex is common, a deviation from the asteroseismic $\log g$ of almost 0.4 dex allows us to discard this line list for the targets analyzed in this work. 


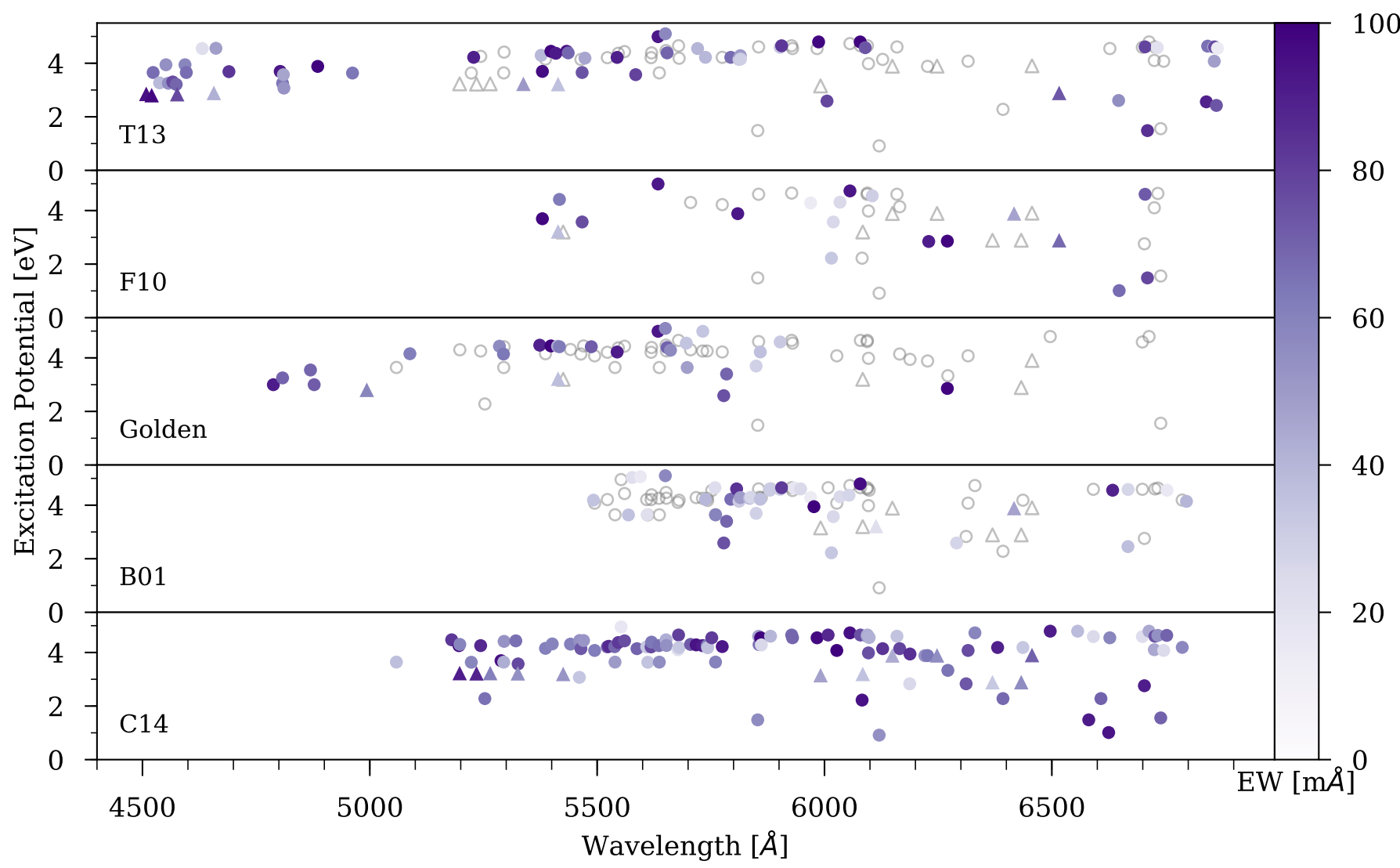

Fig. 4. Excitation potentials for each line list plotted as a function of wavelength. The color coding shows the equivalent widths measured with DAOSPEC. The circles indicate FeI lines and the triangles indicate FeII lines. The gray open circles and triangles in the top four panels represent common lines with the bottom panel.

Other line lists. We tested other line lists as well based on Carraro et al. (2014), Bragaglia et al. (2001), Friel et al. (2010), and Tsantaki et al. (2013); in the following we refer to these works as C14, B01, F10, and T13, respectively. These line lists are all presented in Fig. 4, which shows the excitation potential of all the lines plotted as a function of wavelength color coded by the equivalent width. C14, B01, and F10 are all selected for cool giants, whereas T13 is for cool dwarfs. The lines in common from T13, F10, Golden, and B01 with C14 are plotted with gray open symbols to highlight the differences between the line lists. From this it can be seen that the main difference between these line lists is the wavelength coverage. They all have mostly higher excitation potential lines $(3>\mathrm{eV}>5)$ and all span the entire range in line strengths. A few differences in the line lists stand out, which could potentially trace the differences between the results. The F10 line list has the most even distribution of excitation potentials, which increases the stability of the results for $T_{\text {eff. It does however have significantly fewer lines }}$ than the other line lists, which overall decreases the precision of the results. The B01 and Golden line lists only have one and two lines with excitation potential below $2 \mathrm{eV}$, respectively, which makes the $T_{\text {eff }}$ determination less accurate. Because this has a strong effect on determination of the other parameters, the accuracy on these also decreases. Care should however be taken with strong lines at low excitation potentials as these are more sensitive to $3 \mathrm{D}$ effects (see Bergemann et al. 2012). The amount of FeII lines also has a strong effect on the precision and accuracy of the surface gravity determinations. In these determinations the Golden line list especially stands out, as it has only six FeII lines below $100 \mathrm{~m} \AA$, which could be the explanation for the discrepancy with the asteroseismic surface gravity with this line list. A recent review on the accuracy and precision of stellar parameters is presented in Jofré et al. (2019), where the effects of line selection is also discussed in more detail. Other differences in the obtained atmospheric parameters as a result of line selection could also be issues with the atomic parameters. Even though we ensured all the lines have the best-known atomic parameters, there could still be problems, as the theoretical calculations and laboratory measurements of transitions in iron is very difficult to carry out owing to the complexity of the iron atom. Calibrating each line to the Sun would in principle remove these effects, but this could add new problems because of the differences in atmospheric conditions between the Sun and our stars. This is why it also could be a solution to calibrate to a well-known giant such as Arcturus, but that would also add uncertainties to the analysis due to the uncertainty on the metallicity of Arcturus.

Chosen line list. The results using the different line lists with DAOSPEC are presented in Fig. 5. Based on the surface gravity determined from seismology, the final choice of Fe lines is comprised of the line list from C14 with astrophysical $\log g f$ values based on Grevesse \& Sauval (1998) solar abundances and discarding lines stronger than $\sim 100 \mathrm{~m} \AA$. The choice of non-Fe lines for the final determination of abundances is based on $\mathrm{C} 14$ and Nissen (2015) plus a few additional lines as described in Sect. 3 of Slumstrup et al. (2017). The C14 Fe line list with laboratory oscillator strengths yields a $\log g$ value within $\sim 3 \sigma$ of the asteroseismic value, but with a much higher scatter as shown in Fig. 2 and it was therefore discarded. The F10 line 
list also returns a $\log g$ value close to the asteroseismic value. Nevertheless, this line list has fewer usable absorption lines than the others (42 FeI and FeII lines compared to 109 with C14), which gives significantly larger uncertainties (see Fig. 5) and a lower stability of the analysis (see discussion on this in Sect. 6).

\subsection{Equivalent widths}

The optimal way to measure equivalent widths is to do a manual line by line analysis, for example, using routines such as splot in IRAF. This can however be very time consuming since it is preferable to have many lines for each target and therefore more automatic routines are often used. We tested three different programs that measure equivalent widths in a more automatic way with less user interaction; DAOSPEC (Stetson \& Pancino 2008), ARES (Sousa et al. 2007), and TAME (Kang \& Lee 2012). Comparisons of equivalent width measurements between the three different programs for the M67 target is shown in Fig. 3.

The TAME code can be run both with and without user interaction for the line fitting. We compare the results with a manual user set continuum. When line profile fitting it is possible to choose between Gauss and Voigt profiles, where Voigt profiles should be used for the stronger lines. As mentioned previously, we chose not to work with stronger lines and therefore the comparison is only for lines that can be fitted with a Gauss profile. The TAME program measures equivalent widths 5-7 m $\AA$ higher than DAOSPEC. When using M67 as the test target the measured equivalent widths results in an effective temperature that is higher than that expected from photometry by $130 \mathrm{~K}$ and a surface gravity deviating from that expected from asteroseismology by $\Delta \log g=+0.30$ dex (see Fig. 5). This led us to discard this program.

The ARES program fits lines with a Gaussian profile (for stronger lines, there are other options, but this was not relevant in this case) and does an automatic continuum placement locally around the absorption line aided by a parameter that depends on the $\mathrm{S} / \mathrm{N}$ of the spectrum, which must be set by the user. In Sousa et al. (2008) a table is given with values of this parameter for different $\mathrm{S} / \mathrm{N}$ levels. We varied this parameter to test its effect on the results in the range corresponding to $90<S / N<200$. The impact on the effective temperature was on the order of $\sim 30 \mathrm{~K}$ and on the surface gravity it was $\sim 0.1 \mathrm{dex}$. These are not large variations and we therefore chose to only use the surface gravity as a calibrator due to the much stronger external constraint on this parameter. The result closest to the seismic $\log g$ value was obtained when using the parameter corresponding to a $\mathrm{S} / \mathrm{N}$ between 100 and 125 (the estimated S/N for the M67 spectrum is around 135); the resulting value was however larger than the asteroseismic value by $\Delta \log g=+0.20$ dex.

As mentioned in the previous section and shown in Fig. 3, the scatter on the line strength measurement increases for stronger lines (above $\sim 90 \mathrm{~m} \AA$ ). For the targets in this project this was not very significant as we had enough well-measured weaker lines and therefore did not have to include stronger lines, where perhaps fitting Voigt profiles instead of Gaussians become relevant. In other cases however it can be important to the point that removing the trend between the element abundance versus reduced equivalent width to determine the microturbulence is impossible. The microturbulence will instead have to be calculated from a scaling relation; see, for example, Bruntt et al. (2012). Sousa et al. (2007) did a comparison between DAOSPEC and ARES and found that on average, DAOSPEC measures the equivalent widths $1.9 \mathrm{~m} \AA$ lower than ARES. In our study, the equivalent widths from ARES are 4-6 m $\AA$ higher (left panel of Fig. 3), which is most likely mainly due to continuum placement, as ARES on average places the continuum $\sim 1 \%$ higher than DAOSPEC for our spectrum. There are a variety of reasons why the difference between ARES and DAOSPEC in our analysis is larger than that of Sousa et al. (2007), including different line selection, different spectral types analyzed, or the continuum placement. This is because as mentioned previously, the parameter used by ARES in the continuum placement for our spectrum had to be set for a slightly lower $\mathrm{S} / \mathrm{N}$ than the actual $\mathrm{S} / \mathrm{N}$ of the spectrum.

The final choice of program fell on DAOSPEC, which also fits absorption lines with a Gaussian profile. The continuum is placed automatically around the absorption lines. It was chosen because as shown in Figs. 5 and 6, the measurement of absorption lines yielded results consistent with the more precise and accurate $\log g$ value from asteroseismology. A similar comparison can be performed with the effective temperature from photometry, which shows the same trend as Fig. 6, but with systematically smaller deviations due to the larger uncertainty on the effective temperature from photometry. Even though the photometric effective temperature is less precise than the spectroscopic, it is reassuring that the results showing the best agreement with the asteroseismic $\log g$ are also those in closest agreement with the photometric $T_{\text {eff }}$. This further supports the choice of program and line list.

An additional check of our measured equivalent widths was to apply our method to a solar spectrum. We used a reflected light spectrum of Vesta observed with the same setup as our targets (FIES in the high-resolution mode). We compare our results using DAOSPEC + C14 to solar equivalent widths measurements done by Scott et al. (2015), and found very good agreement on the absorption lines we have in common. The average difference is $-0.4 \mathrm{~m} \AA$ with a scatter of $\sim 3 \mathrm{~m} \AA$. It should be noted that since we have not measured the equivalent widths on the same exact solar spectrum, small differences can be expected. We found a few of our lines to be blended to an amount that makes them questionable to use in the solar case, which led us to discard these lines in our analysis of the giants (N. Grevesse, priv. comm.). After removing these lines, we end up with the following atmospheric parameters for the Sun: $T_{\text {eff }}=5770 \mathrm{~K}, \log g=$ $4.35 \mathrm{dex}, v_{\text {mic }}=0.90 \mathrm{~km} \mathrm{~s}^{-1},[\mathrm{Fe} / \mathrm{H}]=-0.05 \mathrm{dex}$. The small difference to the actual solar atmospheric parameters could arise from the selection of absorption lines, which is tailored to more evolved stars than the Sun.

\section{Atmospheric parameters and abundances}

From the comparisons described in the previous section, we chose an iron line list based on C14 and DAOSPEC to measure the equivalent widths. To calculate atmospheric parameters from the measured equivalent widths we use SPECTRUM (Gray \& Corbally 1994). This routine carries out computations of atmospheric parameters under the assumptions of local thermodynamic equilibrium (LTE) and a plane-parallel atmosphere. The SPECTRUM code comes with auxiliary programs including ABUNDANCE, which is a routine for computing elemental abundances from equivalent widths of individual absorption lines using the COG method (as explained in Sect. 4.1).

One of the required inputs for a spectroscopic analysis is a stellar atmosphere model, where we used a large grid of Kurucz-Castelli ATLAS9 models (Castelli \& Kurucz 2004) interpolated to get the required atmospheric properties. These are 1D plane-parallel model atmospheres that divide the atmosphere 


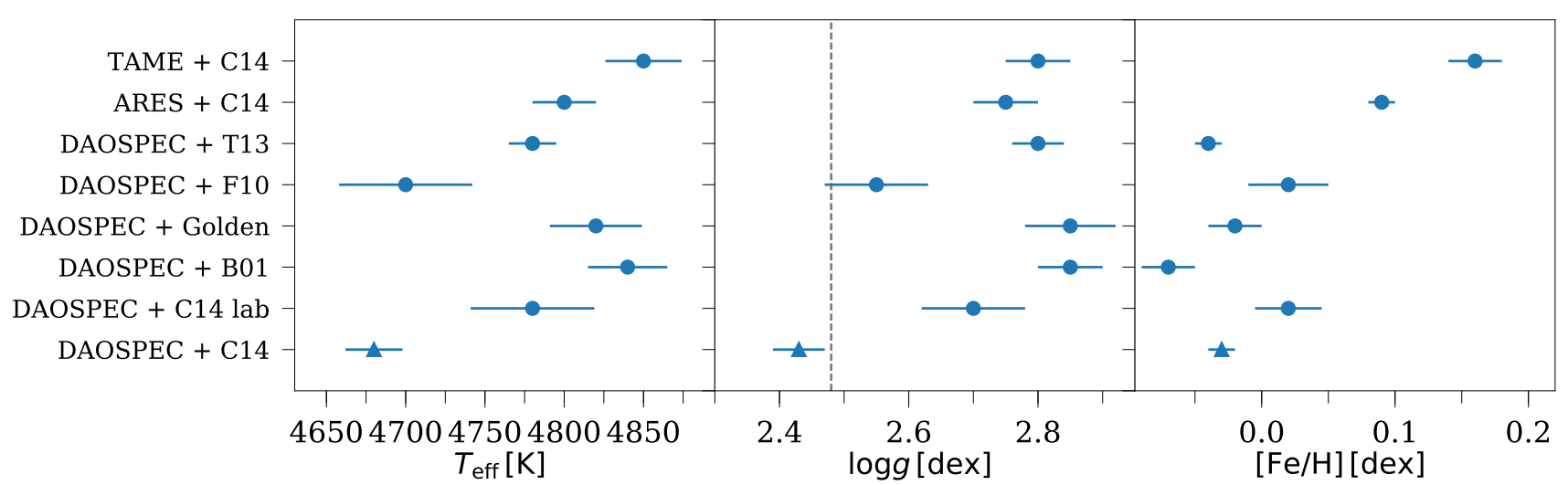

Fig. 5. Comparison of atmospheric parameters for the M67 target with different combinations of line lists and program to measure equivalent widths. Left panel: $T_{\text {eff }}$ variations. Middle panel: $\log g$ variations. Right panel: $[\mathrm{Fe} / \mathrm{H}]$ variations. The final choice of line list and program is shown with a triangle in the bottom row. The dashed line in the middle plot indicates the asteroseismic $\log g$; see Fig. 6 for a comparison.

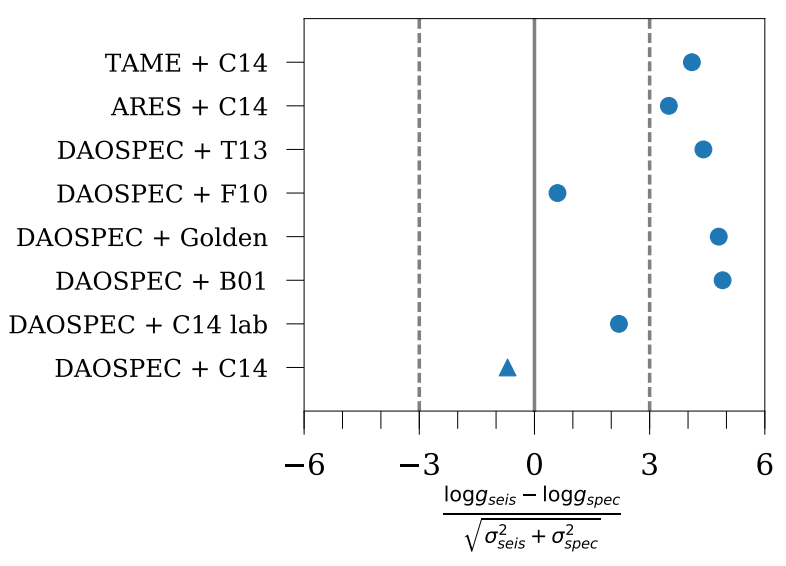

Fig. 6. Deviation from the asteroseismic $\log g$ value for the M67 target for each method combination (middle panel in Fig 5). Full agreement with the asteroseismic $\log g$ is indicated with the vertical line and the two dashed lines show $3 \sigma$ deviations. The final choice of line list and program is shown with a triangle in the bottom row.

of the star into many subsequent layers. Within each layer, the atmosphere is in hydrostatic equilibrium and energy transport is radiative. According to Asplund (2005) and Mashonkina et al. (2011) non-LTE effects are not relevant for FeII lines and mostly relevant for FeI lines in low metallicity stars $([\mathrm{Fe} / \mathrm{H}]<-1.0)$. Thanks to the online tool INSPECT ${ }^{2}$ it is possible to check non-LTE corrections for individual lines given a line strength and atmospheric parameters. The data are from studies by Lind et al. (2012) and Bergemann et al. (2012) and for eight of our FeII and seven of our FeI lines data are available. For these we get average changes in FeII of $<-0.01$ dex and in FeI of $\sim 0.01$ dex. This is within the internal precision of our analysis and therefore we chose not to include nonLTE effects. Three-dimensional LTE effects on spectral line formation was discussed by Collet et al. (2007), where fictitious lines at 3500 and $5000 \AA$ were studied for different stellar atmospheres. For the atmosphere similar to the stars in this study $\left(T_{\text {eff }}=4697 \mathrm{~K}, \log g=2.2 \mathrm{dex}, v_{\text {mic }}=1.5 \mathrm{~km} \mathrm{~s}^{-1}\right.$ and $[\mathrm{Fe} / \mathrm{H}]=0.0)$ the 3D-1D LTE corrections to the Fe abundance for the $5000 \AA$ fictitious line vary between -0.1 and +0.2 dex depending on the excitation potential and equivalent width of the line (see Fig. 5 in Collet et al. 2007). We cannot apply these

\footnotetext{
2 http://www.inspect-stars.com/
}

corrections because they could be anywhere in the range or consistent with zero, but they should however be kept in mind. Since this study, fictitious lines at $6000 \AA$ have been studied in the same way and for our range of atmospheric parameters, the possible changes to the resulting $\mathrm{Fe}$ abundance from these lines are within our uncertainties (R. Collet, priv. comm.). More recently, studies of $3 \mathrm{D}$ non-LTE effects on the iron abundance have been carried out by Amarsi et al. (2016) however only for metal-poor stars $([\mathrm{Fe} / \mathrm{H}]<-1.5 \mathrm{dex})$. The trend found is that the corrections needed to decrease with increasing metallicity (see Fig. 4 in Amarsi et al. 2016). In general we should not extrapolate the results outside the parameter range studied. If however the trend were to continue toward solar metallicity, the corrections needed for our targets would probably be close to or within the internal precision on our result. Another necessary input is a set of atomic and molecular data, where we chose to use the Grevesse \& Sauval (1998) values for the solar atomic abundances. We also tested MARCS atmosphere models, but found very little to no difference in the results with typical changes below $10 \mathrm{~K}, 0.01 \mathrm{dex}$ and $0.01 \mathrm{dex}$ for effective temperature, surface gravity, and metallicity, respectively. This has previously been studied in detail by, for example, Gustafsson et al. (2008) and we do not explore it further here.

The determination of atmospheric parameters was done by requiring that $(1)[\mathrm{Fe} / \mathrm{H}]$ has no systematic dependence on the excitation potential of the FeI lines, i.e., requiring that the atmosphere is in excitation equilibrium; (2) $[\mathrm{Fe} / \mathrm{H}]$ has no systematic dependence on the strength of the FeI lines; and (3) the mean $[\mathrm{Fe} / \mathrm{H}]$ values derived from $\mathrm{FeI}$ and FeII lines are consistent, i.e., requiring the atmosphere to be in ionization equilibrium. The value of $[\mathrm{Fe} / \mathrm{H}]$ as a function of excitation potential is sensitive to the effective temperature and is sensitive to the microturbulence as a function of the reduced equivalent widths of the lines $(\log (\mathrm{EW}) / \lambda)$ because the microturbulence has a larger effect on stronger lines. The surface gravity is determined through its effect on the electron pressure in the stellar atmosphere with the ionization equilibrium between FeI and FeII, as the FeII lines are much more sensitive to pressure changes than the FeI lines. This is however also affected by the effective temperature and metallicity, which makes it necessary to make a number of iterations. For the NGC 6819 and M67 targets, we also calculated a new asteroseismic $\log g$ with the newly found effective temperatures. The possible variation on the effective temperature is however low enough, that the asteroseismic $\log g$ was not significantly affected. The final results for the atmospheric parameters are 
D. Slumstrup et al.: Systematic differences in the spectroscopic analysis of red giants

Table 2. Atmospheric parameters and abundances with only internal uncertainties from spectroscopy with DAOSPEC + C14.

\begin{tabular}{lcccccc}
\hline \hline Target & $T_{\text {eff }}(\mathrm{K})$ & $\log g$ & $v_{\text {mic }}\left(\mathrm{km} \mathrm{s}^{-1}\right)$ & {$[\mathrm{Fe} / \mathrm{H}]$} & $n^{a}$ & {$[\alpha / \mathrm{Fe}]$} \\
\hline NGC 6819-KIC5024327 & $4695 \pm 18$ & $2.52 \pm 0.05$ & $1.19 \pm 0.05$ & $-0.02 \pm 0.01$ & $97 / 12$ & $-0.02 \pm 0.02$ \\
M67-EPIC211415732 & $4680 \pm 18$ & $2.43 \pm 0.04$ & $1.21 \pm 0.05$ & $-0.03 \pm 0.01$ & $97 / 12$ & $0.03 \pm 0.02$ \\
NGC 188-5085 & $4580 \pm 23$ & $2.51 \pm 0.06$ & $1.17 \pm 0.06$ & $0.04 \pm 0.01$ & $95 / 12$ & $0.00 \pm 0.02$ \\
\hline
\end{tabular}

Notes. The analysis presented shows that in a different setup, differences could be up toward $\sim 170 \mathrm{~K}$ for $T_{\text {eff }}, \sim 0.4 \operatorname{dex}$ for $\log g$, and $\sim 0.25$ dex for the metallicity, which should be kept in mind when comparing to other results. ${ }^{(a)}$ Number of FeI/FeII lines used.

presented in Table 2 (all targets) and Fig. 5 (for M67, labeled Daospec + C14).

Metallicity of NGC 6819. As mentioned previously, an asteroseismic value of $\log g$ is also available for the NGC 6819 target, which allows us to use it as a calibrator for our method. When the spectroscopic analysis was carried out for this star with the same setup as for the M67 target, the obtained spectroscopic $\log g(2.52 \mathrm{dex})$ was in excellent agreement with the prediction from asteroseismology (2.55 dex, see Sect. 3). The final $[\mathrm{Fe} / \mathrm{H}]$ for NGC 6819 was found to be $-0.02 \pm 0.01 \mathrm{dex}$, which is on the low end of the previously found values in the literature and in agreement with the result from Lee-Brown et al. $(2015 ;[\mathrm{Fe} / \mathrm{H}]=-0.02 \pm 0.02 \mathrm{dex})$ and slightly lower than that from Bragaglia et al. $(2001 ;[\mathrm{Fe} / \mathrm{H}]=0.09 \pm 0.03 \mathrm{dex})$. As can be seen from Fig. 5 the final combination of Fe line list and program gives results in the lower end of the range obtained for all three atmospheric parameters for the M67 target and this is also the case for the NGC 6819 target. If we instead use the combination of ARES $+\mathrm{C} 14$, we get $[\mathrm{Fe} / \mathrm{H}]=+0.11 \pm 0.01$, which is in better agreement with the high-resolution study from Bragaglia et al. (2001) who did not have asteroseismology available to constrain the surface gravity. We however also discarded this combination for NGC 6819 because the obtained surface gravity is too high $(\log g=2.65)$ to be in agreement with asteroseismology. When doing this comparison it is important to note that within a cluster, the spread in abundances of stars can be on the order of the different results for the NGC 6819 target we find (see, e.g., Liu et al. 2016, for a study on abundance scatter in the Hyades) and since our target is not in either of the two other NGC 6819 studies mentioned in this work, we cannot make a direct comparison.

The individual abundances for all three targets are listed in Table 3. As expected from other studies (Bragaglia et al. 2001; Friel et al. 2010; Önehag et al. 2014) there is no clear sign of alpha enhancement and the abundances are mostly close to solar; there are a few exceptions such as titanium for NGC 6819. The magnesium and yttrium abundances were discussed in more detail in Slumstrup et al. (2017) because it has been shown by, for example, Nissen et al. (2017), that [Y/Mg] is tightly correlated with age for solar-like stars.

\section{Internal uncertainties}

In this paper we discussed possible differences and the effect on stellar atmospheric parameters from choice of line list, atomic parameters, and methods to measure the equivalent widths. On top of these there is the internal precision of the method itself.

When determining $T_{\text {eff }}$ and microturbulence, $v_{\text {mic }}$, the uncertainty on the final zero-slopes of $[\mathrm{Fe} / \mathrm{H}]$ versus excitation potential and $[\mathrm{Fe} / \mathrm{H}]$ versus reduced equivalent width (e.g., Fig. 2) can be easily calculated. To find the internal uncertainty on $T_{\text {eff }}$ and microturbulence, these parameters are varied until a $3 \sigma$
Table 3. Individual abundances.

\begin{tabular}{lcccc}
\hline \hline & NGC 6819 & M67 & NGC 188 & $n^{a}$ \\
\hline$[\mathrm{Na} / \mathrm{Fe}]$ & $0.10 \pm 0.00$ & $0.18 \pm 0.04$ & $0.15 \pm 0.03$ & 2 \\
{$[\mathrm{Mg} / \mathrm{Fe}]$} & $-0.05 \pm 0.03$ & $0.06 \pm 0.03$ & $0.04 \pm 0.04$ & 4 \\
{$[\mathrm{Al} / \mathrm{Fe}]$} & $0.02 \pm 0.05$ & $0.11 \pm 0.06$ & $0.14 \pm 0.07$ & 2 \\
{$[\mathrm{Si} / \mathrm{Fe}]$} & $0.09 \pm 0.04$ & $0.14 \pm 0.04$ & $0.14 \pm 0.04$ & 14 \\
{$[\mathrm{Ca} / \mathrm{Fe}]$} & $-0.11 \pm 0.06$ & $-0.01 \pm 0.05$ & $-0.09 \pm 0.08$ & 7 \\
{$[\mathrm{Ti} / \mathrm{Fe}]$} & $-0.24 \pm 0.05$ & $-0.11 \pm 0.03$ & $-0.12 \pm 0.04$ & 19 \\
{$[\mathrm{Cr} / \mathrm{Fe}]$} & $-0.08 \pm 0.04$ & $-0.02 \pm 0.04$ & $-0.03 \pm 0.05$ & 18 \\
{$[\mathrm{Ni} / \mathrm{Fe}]$} & $0.00 \pm 0.03$ & $0.09 \pm 0.03$ & $0.12 \pm 0.03$ & 32 \\
{$[\mathrm{Zn} / \mathrm{Fe}]$} & $-0.17 \pm 0.04$ & $-0.07 \pm 0.14$ & $-0.11 \pm 0.19$ & 2 \\
{$[\mathrm{Y} / \mathrm{Fe}]$} & $0.03 \pm 0.04$ & $0.07 \pm 0.05$ & $-0.02 \pm 0.06$ & 3 \\
\hline
\end{tabular}

Notes. ${ }^{(a)}$ Number of absorption lines used in the determination.

difference is produced in one of the two slopes or in the difference between FeI and FeII abundances. The change in $T_{\text {eff }}$ or microturbulence is then divided by the highest produced difference to give the final $1 \sigma$ uncertainty, which corresponds to the internal uncertainty for these parameters listed in Table 2 .

To calculate the internal $\log g$ uncertainty we can use the difference between FeI and FeII abundances determined as $\Delta[\mathrm{Fe} / \mathrm{H}] / \sigma_{\Delta[\mathrm{Fe} / \mathrm{H}]}$, where

$\sigma_{\Delta[\mathrm{Fe} / \mathrm{H}]}=\sqrt{\left(\frac{\sigma_{\mathrm{FeI}}}{\sqrt{n_{\mathrm{FeI}}-1}}\right)^{2}+\left(\frac{\sigma_{\mathrm{FeII}}}{\sqrt{n_{\mathrm{FeII}}-1}}\right)^{2}}$.

In this case, $\sigma_{\mathrm{FeI}, I I}$ is the RMS scatter on the measured abundances of each ionization stage and $n_{\text {FeI,II }}$ is the amount of lines used for the measurements. Once again, we vary $\log g$ until a $3 \sigma$ difference is produced in one of the two slopes or in the difference between FeI and FeII abundances. The internal uncertainty on $[\mathrm{Fe} / \mathrm{H}]$ listed in Table 2 corresponds to the standard error of the mean.

Another way of characterizing the internal uncertainties is to test the stability of the results. This can be done with a sensitivity analysis, which is a test of how sensitive the final metallicity is to changes in the other atmospheric parameters. This was carried out for the M67 target and the results are presented in Table 4. It was only done for one target because the quality of the spectra for each star and their atmospheric parameters are so similar that the sensitivity can be assumed to be almost the same for all of them. Comparison of these results to the internal uncertainties in Table 2 reveals that the stability of the analysis is high because the internal uncertainties are much lower than the variation in the atmospheric parameters in the sensitivity analysis.

On top of the internal uncertainties described in this section, there is significant scatter arising from using different approaches (e.g., line lists and measurements of equivalent 
Table 4. Sensitivity of the metallicity to the other atmospheric parameters for the M67 target.

\begin{tabular}{cccc}
\hline \hline & $+100 \mathrm{~K} T_{\text {eff }}$ & $+0.1 \mathrm{dex} \log g$ & $+0.1 \mathrm{~km} \mathrm{~s}^{-1} v_{\text {mic }}$ \\
\hline$\Delta[\mathrm{Fe} / \mathrm{H}]$ & $+0.03 \mathrm{dex}$ & $+0.02 \mathrm{dex}$ & $-0.03 \mathrm{dex}$ \\
\hline
\end{tabular}

widths) as discussed in this paper. This is often not characterized, but instead an extra 0.1 dex is simply added to the internal uncertainty on $[\mathrm{Fe} / \mathrm{H}]$ and $0.1-0.2$ dex for $\log g$, while a systematic uncertainty of about $100 \mathrm{~K}$ is typically added for the effective temperature (see, e.g., Smalley 2005; Bruntt et al. 2010, 2012). Our analysis shows that the differences can be much larger than this; Fig. 5 shows $\sim 170 \mathrm{~K}$ for $T_{\text {eff }}, \sim 0.4$ dex for $\log g$, and $\sim 0.25$ dex for the metallicity. This should be kept in mind when comparing to other results that make use of a different combination of line list, program for measuring line strengths, and atomic data.

\section{Conclusions}

We presented a detailed spectroscopic study of three targets in three open clusters, NGC 6819, M67, and NGC 188. These targets are all in the same evolutionary stage and observed with the exact same instrumental setup. To our knowledge the data for the NGC 6819 and NGC 188 targets are of higher quality (higher resolution and higher $\mathrm{S} / \mathrm{N}$ ) than previously studied as described in the introduction. Along with this, we had asteroseismic data available for the two targets in NGC 6819 and M67, which allowed us to use the M67 target as a benchmark in the analysis and the NGC 6819 target to show the method was properly calibrated. In turn, it helped us to choose between different combinations of line lists and equivalent width measurement tools. Testing the different line lists and programs for measuring equivalent widths allowed us to characterize systematic differences within the method of a line by line spectroscopic analysis utilizing excitation and ionization equilibria in the stellar atmosphere.

The differences found on the atmospheric parameters for the M67 target with the different combinations were shown in Fig. 5, where the scatter found in effective temperature is $\sim 170 \mathrm{~K}, \sim 0.4$ dex in surface gravity, and $\sim 0.25$ dex in metallicity. The spread on the results for surface gravity is particularly high compared to the internal uncertainty obtained by each combination of line list and equivalent width measurement program. Asteroseismology allowed us to choose the most robust option for this type of targets, which was the combination of DAOSPEC and the line list based on C14. This was also the result in closest agreement with the effective temperature from photometry.

In the current era of large spectroscopic surveys, this study highlights the possible pitfalls still existing in high precision spectroscopic analysis and why it is of crucial importance to have external constraints to calibrate the results to achieve high accuracy.

Using this setup, we also established the metallicity of NGC 6819, which is an old open cluster in the Kepler field, to be close to solar, i.e., $[\mathrm{Fe} / \mathrm{H}]=-0.02 \pm 0.01$ dex. This result is on the lower end of previous determinations of the iron abundance for this cluster (see, e.g., Bragaglia et al. 2001; Lee-Brown et al. 2015), and it highlights the importance of carefully selecting and measuring adequate absorption lines with good atomic data for a specific set of targets.
Acknowledgements. We thank the anonymous referee for her/his careful revision of the manuscript and constructive comments that helped improve the quality of the paper. We thank Remo Collet for fruitful discussion on 3D effects. We thank Mikkel N. Lund for help with the figures. This work has made use of the VALD database, operated at Uppsala University, the Institute of Astronomy RAS in Moscow, and the University of Vienna. This research has made use of the SIMBAD database, operated at CDS, Strasbourg, France. Funding for the Stellar Astrophysics Centre is provided by The Danish National Research Foundation (Grant DNRF106). V.S.A. acknowledges support from VILLUM FONDEN (research grant 10118) and the Independent Research Fund Denmark (Research grant 7027-00096B).

\section{References}

Amarsi, A. M., Lind, K., Asplund, M., Barklem, P. S., \& Collet, R. 2016, MNRAS, 463, 1518

Asplund, M. 2005, ARA\&A, 43, 481

Bergemann, M., Lind, K., Collet, R., Magic, Z., \& Asplund, M. 2012, MNRAS, 427, 27

Borucki, W. J., Koch, D. G., Dunham, E. W., \& Jenkins, J. M. 1997, in Planets Beyond Solar System and the Next Generation of Space Mission, ed. D. R. Soderblom (San Francisco: ASP), 21

Bragaglia, A., Carretta, E., Gratton, R., et al. 2001, AJ, 121, 327

Brogaard, K., VandenBerg, D. A., Bruntt, H., et al. 2012, A\&A, 543, A106

Brogaard, K., Hansen, C. J., Miglio, A., et al. 2018, MNRAS, 476, 3729

Brown, T. M., Gilliland, R. L., Noyes, R. W., \& Ramsey, L. W. 1991, ApJ, 368, 599

Bruntt, H., Bedding, T. R., Quirion, P. O., et al. 2010, MNRAS, 405, 1907

Bruntt, H., Frandsen, S., \& Thygesen, A. O. 2011, A\&A, 528, A121

Bruntt, H., Basu, S., Smalley, B., et al. 2012, MNRAS, 423, 122

Carraro, G., Villanova, S., Monaco, L., et al. 2014, A\&A, 562, A39

Casagrande, L., Aguirre, V. S., Stello, D., et al. 2014, ApJ, 787, 110

Casagrande, L., Silva Aguirre, V., Schlesinger, K. J., et al. 2016, MNRAS, 455, 987

Casamiquela, L., Carrera, R., Blanco-Cuaresma, S., et al. 2017, MNRAS, 470, 4363

Castelli, F., \& Kurucz, R. L. 2004, ArXiv e-prints [arXiv:0405087]

Collet, R., Asplund, M., \& Trampedach, R. 2007, A\&A, 469, 687

Corsaro, E., Stello, D., Huber, D., et al. 2012, ApJ, 757, 190

De Silva, G. M., Freeman, K. C., Bland-Hawthorn, J., et al. 2015, MNRAS, 449, 2604

Doyle, A. P., Smalley, B., Faedi, F., Pollacco, D., \& Gómez Maqueo Chew, Y. 2017, MNRAS, 469, 4850

Friel, E. D., Jacobson, H. R., \& Pilachowski, C. A. 2010, AJ, 139, 1942

García Pérez, A. E., Allende Prieto, C., Holtzman, J. A., et al. 2016, AJ, 151, 144

Gilmore, G., Randich, S., Asplund, M., et al. 2012, The Messenger, 147, 25

Gray, R. O., \& Corbally, C. J. 1994, AJ, 107, 742

Grevesse, N., \& Sauval, A. J. 1998, Space Sci. Rev., 85, 161

Gustafsson, B., Edvardsson, B., Eriksson, K., et al. 2008, A\&A, 486, 951

Handberg, R., Brogaard, K., Miglio, A., et al. 2017, MNRAS, 472, 979

Hawkins, K., Masseron, T., Jofré, P., et al. 2016, A\&A, 594, A43

Heiter, U., Jofré, P., Gustafsson, B., et al. 2015, A\&A, 49, A1

Hinkel, N. R., Young, P. A., Pagano, M. D., et al. 2016, ApJS, 226, 1

Hole, K. T., Geller, A. M., Mathieu, R. D., et al. 2009, AJ, 138, 159

Howell, S. B., Sobeck, C., Haas, M., et al. 2014, PASP, 126, 398

Huber, D., Ireland, M. J., Bedding, T. R., et al. 2012, ApJ, 760, 32

Huber, D., Zinn, J., Bojsen-Hansen, M., et al. 2017, ApJ, 844, 102

Jofré, P., Heiter, U., Soubiran, C., et al. 2014, A\&A, 564, A133

Jofré, P., Heiter, U., \& Soubiran, C. 2019, ARA\&A, in press [arXiv: 1811.08041]

Kang, W., \& Lee, S.-G. 2012, MNRAS, 425, 3162

Kjeldsen, H., \& Bedding, T. R. 1995, A\&A, 293, 87

Lebzelter, T., Heiter, U., Abia, C., et al. 2012, A\&A, 547, A108

Lee-Brown, D. B., Anthony-Twarog, B. J., Deliyannis, C. P., Rich, E., \& Twarog, B. A. 2015, AJ, 149, 121

Lind, K., Bergemann, M., \& Asplund, M. 2012, MNRAS, 427, 50

Liu, F., Yong, D., Asplund, M., Ramírez, I., \& Meléndez, J. 2016, MNRAS, 457, 3934

Majewski, S. R., Schiavon, R. P., Frinchaboy, P. M., et al. 2017, AJ, 154, 94

Mashonkina, L. I., Gehren, T., Shi, J.-R., Korn, A. J., \& Grupp, F. 2011, A\&A, 528, A87

Meibom, S., Grundahl, F., Clausen, J. V., et al. 2009, AJ, 137, 5086

Miglio, A., Brogaard, K., Stello, D., et al. 2012, MNRAS, 419, 2077

Miglio, A., Chiappini, C., Morel, T., et al. 2013, MNRAS, 429, 423

Miglio, A., Chaplin, W. J., Brogaard, K., et al. 2016, MNRAS, 461, 760

Nissen, P. E. 2015, A\&A, 579, A52

Nissen, P. E., Høg, E., \& Schuster, W. J. 1997, ESA SP, 225 
Nissen, P. E., Silva Aguirre, V., Christensen-Dalsgaard, J., et al. 2017, A\&A, 608, A112

Önehag, A., Gustafsson, B., \& Korn, A. J. 2014, A\&A, 562, A102

Pinsonneault, M. H., Elsworth, Y., Epstein, C., et al. 2014, ApJS, 215, 19

Pinsonneault, M. H., Elsworth, Y. P., Tayar, J., et al. 2018, ApJS, 239, 32

Piskunov, N. E., Kupka, F., Ryabchikova, T. a., Weiss, W. W., \& Jeffery, C. S. 1995, A\&AS, 112, 525

Ramírez, I., \& Meléndez, J. 2005, ApJ, 626, 465

Ramírez, I., Meléndez, J., Bean, J., et al. 2014, A\&A, 572, A48

Randich, S., Sestito, P., \& Pallavicini, R. 2003, A\&A, 399, 133

Randich, S., Gilmore, G., \& Consortium, G.-E. 2013, The Messenger, 154, 47

Rosvick, J. M., \& VandenBerg, D. A. 1998, AJ, 115, 1516

Sahlholdt, C. L., Silva Aguirre, V., Casagrande, L., Mosumgaard, J. R., \& Bojsen-Hansen, M. 2018, MNRAS, 476, 1931

Scott, P., Asplund, M., Grevesse, N., Bergemann, M., \& Jacques Sauval, A. 2015, A\&A, 573, A26

Silva Aguirre, V., Casagrande, L., Basu, S., et al. 2012, ApJ, 757, 99

Silva Aguirre, V., Lund, M. N., Antia, H. M., et al. 2016, ApJ, 835, 1

Silva Aguirre, V., Bojsen-Hansen, M., Slumstrup, D., et al. 2018, MNRAS, 5500, 5487

Skrutskie, M. F., Cutri, R. M., Stiening, R., et al. 2006, AJ, 131, 1163
Slumstrup, D., Grundahl, F., Brogaard, K., et al. 2017, A\&A, 604, L8 Smalley, B. 2005, Mem. Soc. Astron. It., 8, 130

Smiljanic, R., Korn, A. J., Bergemann, M., et al. 2014, A\&A, 570, A122

Sneden, C. 1973, Ph.D. Thesis, The University of Texas at Austin, USA

Sousa, S. G., Santos, N. C., Israelian, G., Mayor, M., \& Monteiro, M. J. P. F. G. 2007, A\&A, 469, 783

Sousa, S. G., Santos, N. C., Mayor, M., et al. 2008, A\&A, 487, 373

Steinmetz, M., Zwitter, T., Siebert, A., et al. 2006, AJ, 132, 1645

Stello, D., Vanderburg, A., Casagrande, L., et al. 2016, ApJ, 832, 133

Stetson, P. B., \& Pancino, E. 2008, PASP, 120, 1332

Stetson, P. B., McClure, R. D., \& VandenBerg, D. A. 2004, PASP, 116, 1012

Taylor, B. J. 2007, AJ, 133, 370

Thygesen, A. O., Frandsen, S., Bruntt, H., et al. 2012, A\&A, 160, A1

Torres, G. 2010, AJ, 140, 1158

Tsantaki, M., Sousa, S. G., Adibekyan, V. Z., et al. 2013, A\&A, 555, A150

Valenti, J. A., \& Piskunov, N. 1996, A\&AS, 118, 595

Valentini, M., Chiappini, C., Miglio, A., et al. 2016, Astron. Nachr., 337, 970

White, T. R., Huber, D., Maestro, V., et al. 2013, MNRAS, 433, 1262

Yadav, R. K. S., Bedin, L. R., Piotto, G., et al. 2008, A\&A, 484, 609

\section{Appendix A: Line list}

Table A.1. Line list.

\begin{tabular}{|c|c|c|c|c|c|c|}
\hline $\begin{array}{l}\text { Wavelength } \\
\text { (§) }\end{array}$ & Element & $\begin{array}{l}\text { Exc. Pot. } \\
(\mathrm{eV})\end{array}$ & $\log g f$ & NGC 6819-KIC5024327 & $\begin{array}{l}\text { EW (mA) } \\
\text { M67-EPIC211415732 }\end{array}$ & NGC 188-5085 \\
\hline 5052.1350 & 6.0 & 7.685 & -1.433 & 12.0 & 10.3 & 11.1 \\
\hline 6154.2170 & 11.0 & 2.102 & -1.600 & 81.5 & 82.1 & 92.7 \\
\hline 6160.7420 & 11.0 & 2.104 & -1.260 & 105.4 & 107.6 & 111.4 \\
\hline 5711.0910 & 12.0 & 4.346 & -1.742 & 129.6 & 133.7 & 138.9 \\
\hline 6318.7010 & 12.0 & 5.108 & -2.020 & 68.0 & 71.3 & 77.3 \\
\hline$\ldots$ & $\ldots$ & $\ldots$ & $\ldots$ & $\ldots$ & $\ldots$ & $\ldots$ \\
\hline
\end{tabular}

Notes. The full table is available at the CDS. 